\title{
Atypical Cutaneous Lymphoproliferative Disorder: A Fatal Mimic of Cutaneous T-Cell Lymphoma in a Patient with HIV Infection
}

\author{
Veronica Nguyen $^{1}$, Russell Dorer ${ }^{2}$, David M. Aboulafia ${ }^{3,4^{*}}$ \\ ${ }^{1}$ Albany Medical College, Albany, USA; ${ }^{2}$ Division of Pathology, Virginia Mason Medical Center, Seattle, USA; ${ }^{3}$ Division of Hema- \\ tology and Oncology, Virginia Mason Medical Center, Seattle, USA; ${ }^{4}$ Division of Hematology, University of Washington, Seattle, \\ USA. \\ Email: *hemdma@vmmc.org
}

Received November $14^{\text {th }}, 2012$; revised December $16^{\text {th }}$, 2012; accepted December $28^{\text {th }}, 2012$

\begin{abstract}
Atypical cutaneous lymphoproliferative disorder (ACLD) is a rare condition that has been associated with HIV infection. Patients with ACLD present with diffuse, erythematous and pruritic skin lesions accompanied by generalized lymphadenopathy. The clinical characteristics of ACLD overlap most notably with several other conditions including Mycosis Fungoides/Sézary Syndrome (MF/SS), a cutaneous lymphoma of T-cell lineage. Unlike Mycosis Fungoides, the noxious infiltrates of ACLD are not monoclonal but polyclonal and consist of cytotoxic CD8+ T-cells instead of CD4+ T-cells or B-cells. Highly active antiretroviral therapy (HAART) has been reported to improve ACLD. We describe the case of a Caucasian man with longstanding HIV infection who presented with severe erythroderma. Skin and lymph node biopsies showed polyclonal CD8+ T-cell infiltrates. Gene rearrangement studies did not reveal an obvious clonal disorder. Hallmark peripheral blood findings consisting of a severe depletion of CD4+ T-lymphocytes and markedly elevated CD8+ cells provided an important diagnostic clue. Despite the purported benefits of HAART in ameliorating this disorder, erythroderma and extreme pruritus improved only after the patient began taking mycophenolate mofetil and hydroxyurea. Unfortunately, he succumbed to complications of methicillin-resistant Staphylococcus aureus septicemia. We alert readers to this rare HIV-associated condition which may mimic other benign and malignant skin conditions and briefly discuss diagnostic and therapeutic options.
\end{abstract}

Keywords: Atypical Cutaneous Lymphoproliferative Disorder; Mimic Fatal; Cutaneous T-Cell Lymphoma; HIV Infection

\section{Introduction}

Atypical cutaneous lymphoproliferative disorder (ACLD) is a rare but potentially life-threatening complication of HIV infection. It most commonly manifests as diffuse erythroderma due to massive infiltration of polyclonal CD8+ T-cells involving the dermis and epidermis [1]. First described by Longacre and colleagues in 1989, ACLD has been reported under a number of other pseudonyms including HIV-associated cutaneous CD8+ pseudolymphoma, pseudo-Sézary syndrome and Mycosis Fungoides (MF)-like lesions [2,3]. When CD8+ polyclonal cells involve other organs, most commonly the parotids, but also the lungs, liver, gastrointestinal tract and kidneys, it is referred to as diffuse infiltrative lymphocytosis syndrome (DILS) [4]. When DILS is restricted to the head and neck, it manifests as bilateral

*Corresponding author. parotid gland enlargement with Sicca syndrome and associated lymphadenopathy. The lung is the most common extraglandular site of DILS, and patients with pulmonary involvement often present with fever, shortness of breath and interstitial infiltrates which, in the absence of appropriate immunostains from sputum or pulmonary lavage, can be mistaken for Pneumocystis Jiroveci pneumonia [5]. DILS can also present as a polyneuropathy that can include both cervical and lumbosacral nerves [6]. A tipoff that the patient may have ACLD or DILS is peripheral blood lymphocyte flow cytometry abnormalities showing depletion of CD4+ T-cells and a striking increase in CD8+ T-cells in the setting of a normal or increased total lymphocyte count [4].

Prior to the advent of HAART, symptoms of individuals with ACLD or DILS rarely improved [3,7]. Systemic multiagent anti-lymphoma chemotherapy, antivirals, oral, topical and systemic corticosteroids and oral ketocona- 
zole have all been used in an attempt to treat this condition, but none of these strategies proved clearly successful in the pre-HAART era [2,3]. More recently, small case series and isolated case reports have suggested that HAART significantly improves the erythroderma and end-organ dysfunction associated with ACLD and that its use has led to a decreased incidence of this condition $[2,7]$.

We describe a patient with HIV infection who presented with severe pruritus and diffuse erythroderma, weight loss, and palpable and generalized lymphadenopathy. The clinical presentation was disconcerting for MF, but assorted biopsies showed only a polyclonal expansion of CD8+ lymphocytes infiltrating the dermis, epidermis and lymph nodes and no clonal gene rearrangements. The specter of DILS was heightened when flow cytometry of peripheral blood lymphocytes revealed a profound reversal of the CD4+:CD8+ cell ratio with a marked expansion of CD8+ cells. Despite receiving HAART, the patient succumbed to complications of methicillin-resistant Staphylococcus aureus (MRSA) septicemia. Herein, we alert readers to the hallmarks of this unusual condition and review mimics that can be confused with ACLD.

\section{Case Report}

In 1989, a 43-year-old Caucasian man who had sex with other men tested HIV seropositive and subsequently received zidovudine monotherapy. When HAART became readily available in 1996, he was switched to a regimen consisting of stavudine, lamivudine and nevirapine. Over the ensuing years, he had a consistently non-detectable HIV viral load, and he continued to work full time as a registered nurse. His CD4+ count remained low, typically hovering between $60-110$ cells/uL.

The patient sought medical attention for a nonspecific lower extremity skin rash which was treated with topical triamcinolone with benefit. Six months later, he was again seen by a dermatologist after he noted progressive facial erythroderma. When the rash failed to improve despite the implementation of various topical agents and the cessation of prophylactic trimethoprim-sulfamethoxazole, a series of skin biopsies were obtained. These showed features of epidermal hyperplasia with elongated and thickened rete pegs, confluent parakeratosis and small collections of neutrophils within the stratum corneum and moderately dense, superficial perivascular lymphocytic inflammatory infiltrates (Figure 1). Immunofluorescence studies did not reveal a clonal disorder. Subsequent computerized tomograms of the neck, chest, abdomen and pelvis showed modest adenopathy involving the left axilla and right groin. There were no pulmonary abnormalities.
Over the next several months, the patient received topical and oral corticosteroids, oral retinoic acid, oral methotrexate, as well as intravenous infliximab. However, none of these interventions led to significant or consistent benefit. The patient temporarily stopped HAART for three weeks to see if this strategy would curtail the erythroderma which had now progressed to involve his trunk and extremities. There was no significant improvement in the degree of pruritus or in the thickness or erythema of his skin, and thus, he restarted HAART with efavirenz substituted for nevirapine.

He was referred to medical oncology and hematology for additional evaluation after losing seven kilograms over the ensuing three months. He described severe and unrelenting pruritus which prevented him from sleeping despite multiple trials with sleep medications, anxiolytics, antihistamines and an anti-psychotic agent. He did not experience night sweats or fevers except when he contracted MRSA cellulitis of the hand. With oral antibiotics, the infection and fevers resolved. His physical exam was notable for proximal muscle atrophy and diffuse erythroderma most prominent on the nape of neck, trunk and feet and without preference for light-exposed areas (Figures 2(a)-(c)). Multiple excoriations and scabbed lesions involved all four extremities, but he was without overt cellulitis or skin desquamation. There was no visceromegaly and only modest axillary and groin lymphadenopathy. Laboratory studies included a white blood count (WBC) of 23,000 with hematocrit of $42 \%$ and platelet count of 326,000. Despite the use of steroids (50 mg daily), the WBC was comprised principally of lymphocytes. His chemistry and liver function tests were

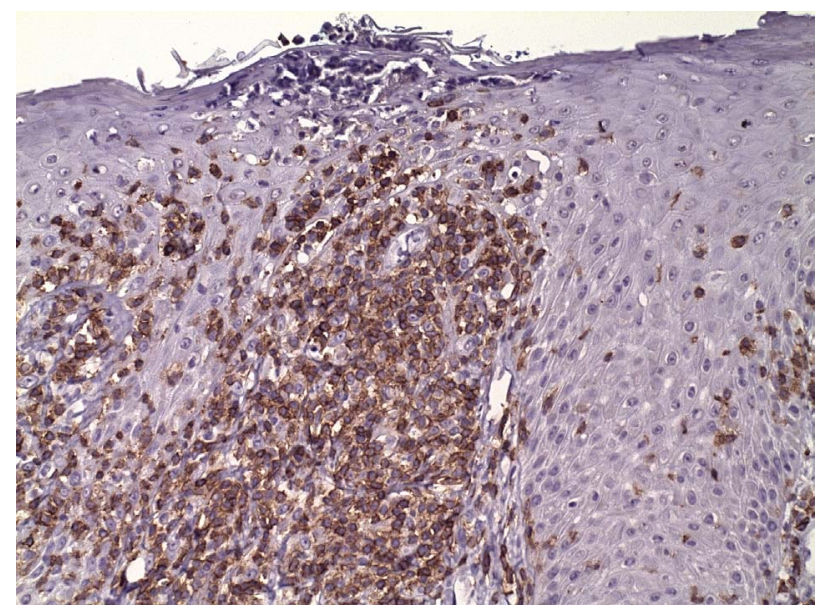

Figure 1. Histological sections of epidermal biopsy show features of hyperplasia with elongated and thickened rete pegs, confluent parakeratosis and small collections of neutrophils within the stratum corneum and moderately dense, superficial perivascular lymphocytic inflammatory infiltrate. Figure 1 shows CD8+ lymphocytic infiltrates with Hemotoxylin and Eosin stain. 

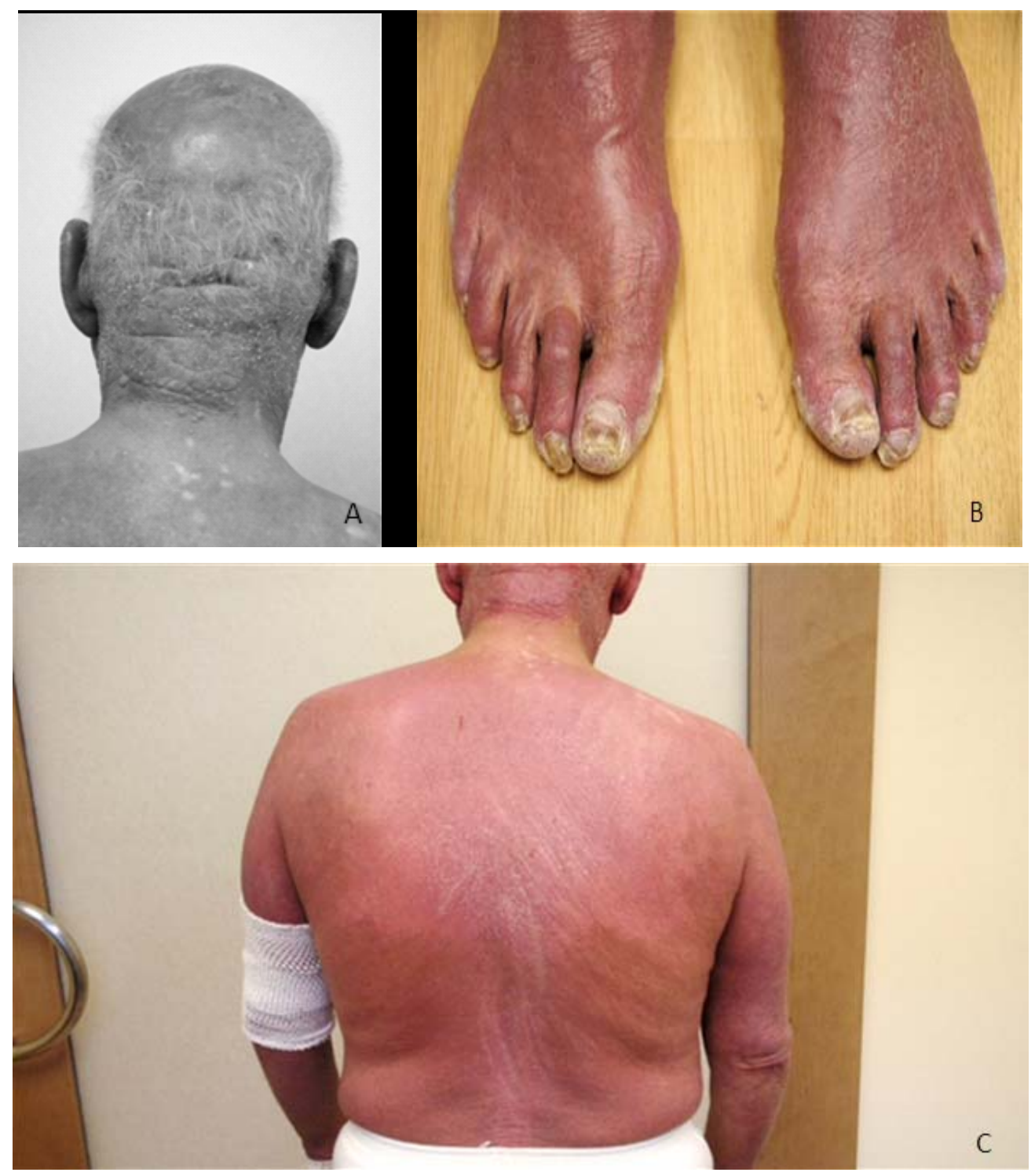

Figure 2. (a)-(c) Clinical features in patient presented with dry and thickened skin with diffuse, pigmented, pruriginous erythroderma and desquamation most prominent on the nape of neck, feet and back.

within normal limits, but a serum lactate dehydrogenase was $347 \mathrm{IU} / \mathrm{L}$ (normal < 252). A serum protein electrophoresis did not reveal a monoclonal band. When checked for the first time in six months, his CD4+ count had increased dramatically to 598 cells/uL and his CD8+ count was profoundly elevated to 13,333 cells/uL with a CD4:CD8 ratio that was markedly inverted at .045 (normal, 1.7). His HIV-1 RNA viral load remained non-detectable at less than 75 copies $/ \mathrm{mL}$. He was advised to taper his corticosteroids and begin hydroxyurea at a dose of $1000 \mathrm{mg}$ daily.

One month later, the patient was hospitalized with MRSA pneumonia. Shortly after his infection resolved, mycophenolate mofetil, $1000 \mathrm{mg}$ twice daily, was added to his regimen of hydroxyurea. Over the next several weeks, his skin began to improve markedly with less erythroderma and less keratosis and ulceration. Two months later, his CD4+ count had dropped from a zenith of 1035 to 295 cells/uL and his CD8+ count decreased to 1601 cells/uL. While recuperating in a skilled nursing facility, he became acutely hypotensive and febrile. Blood cultures grew out MRSA. His condition rapidly deteriorated, and his request to be transitioned to strict comfort care was honored. He died shortly thereafter and an autopsy was not performed.

\section{Discussion}

Defining characteristics of ACLD in patients with im- 
munosuppression are diffuse erythrodermatous plaques comprised of polyclonal CD8+ predominant lymphocytes which most often involve skin, accompanied by generalized lymphadenopathy, severe pruritus and depletion of peripheral blood CD4+ lymphocytes. These findings are in contrast to DILS where cutaneous disease is not common but a proclivity to involve the parotid glands is to be expected. The incidence of HIV-associated DILS is low, $3 \%$ in the United States [8]. The incidence of ACLD is even rarer, with only 39 reported cases of individuals with HIV described in the medical literature since 1992 [5].

The differential diagnosis of pruritus and diffuse erythroderma in a patient with HIV infection is broad and may include scabies, papular pruritic eruption of HIV, eosinophilic pustular folliculitis and drug reactions [9-13]. In the context of weight loss, mild adenopathy, and absence of a drug reaction or an infection, the patient was referred to medical hematology and oncology to rule out a paraneoplastic skin condition, MF or an alternative cutaneous T-cell lymphoma (CTCL), including Sézary's syndrome and adult T-cell leukemia/lymphoma (ATLL). With each of these conditions, symptoms of fever, night sweats and weight loss may be prominent [14-18]. In addition, generalized erythroderma, lymphadenopathy, atypical T-cells in peripheral blood and hepatosplenomegaly may dominate the clinical presentation with each of these conditions. However, upon further inspection of the clonal origins of the T-cells in MF showcases resident effector memory T-cells. Sézary syndrome is associated with central memory T-cells and ATLL is associated with mature helper T-cells. All of these cells are CD4+ lymphocytes [19-21]. In contrast, with ACLD, representative biopsies of involved skin and lymph nodes show that the infiltrates are comprised of mostly cytotoxic CD8+ T-cells [22-24] (Table 1).

The pathogenesis of ACLD has not been fully characterized. However, a possible explanation of this condition isthe expansion of cytotoxic CD8+ T-cells may be related to the diminished numbers of circulating CD4+ Tcells and the reciprocal regulatory processes between the two different cell types [2]. When there is marked depletion of CD4+ T-cells and a large increase in CD8+ Tcells, the regulatory balance is no longer intact and uncontrolled cytotoxic CD8+ cell expansion may occur $[2,25]$. Remission of skin symptoms after HAART initiation suggests the reduction of HIV viral load and the reconstitution of CD4+ T-lymphocytes can exert regulatory effects on the expansion of CD8+ cells [2]. Thus, ACLD is not driven by a malignant condition, but rather, it is a disease that is reactive in nature and can improve secondary to HAART effects on HIV viral load [2]. The factors that further lead to the migration of CD8+ cells to involve the dermis, however, remain unexplained.

There is no clear treatment for ACLD save HAART. In the eight cases reported by Sbidian and colleagues and the one case described by Egbers and colleagues, the earliest response occurred within two weeks of initiation of HAART, and the median time to maximal response was five months after exposure to HAART [2,5]. It is important that the hallmarks of ACLD are recognized and the diagnosis is made early in the course of the disease to minimize suffering and potentially fatal infections [9]. Of the reported cases, more than half of the patients died within one year of initial presentation of ACLD, but the causes of death were secondary to other complications of AIDS; none died directly from this condition or from malignancy [5]. Our patient was unique. He had long been on effective HAART as evidenced by a consistently non-detectable HIV viral load when he was diagnosed with ACLD with an elevated CD4+ count which may be due to an aberrant immune reconstitution. He showed signs of improvement only after he received cytoreductive (hydroxyurea) and immunosuppressive (mycophenolate mofetil) treatment and while corticosteroids were in the process of being weaned.

Table 1. Comparison between ACLD and other malignant diseases that are associated with infiltrating lymphocytes, lymphadenopathy and erythroderma and treatment.

\begin{tabular}{|c|c|c|c|c|c|}
\hline Variables & ACLD & DILS & CTCL: MF & CTCL: SS & ATLL \\
\hline $\begin{array}{l}\text { Enlargement of } \\
\text { glands or organs }\end{array}$ & $\begin{array}{l}\text { General } \\
\text { lymphadenopathy }\end{array}$ & $\begin{array}{l}\text { Bilateral parotid } \\
\text { enlargements, } \\
\text { generalized } \\
\text { lymphadenopathy }\end{array}$ & $\begin{array}{l}\text { Hepatosplenomegaly, } \\
\text { generalized } \\
\text { lymphadenopathy }\end{array}$ & $\begin{array}{l}\text { Generalized } \\
\text { lymphadenopathy }\end{array}$ & $\begin{array}{l}\text { Generalized } \\
\text { lymphadenopathy }\end{array}$ \\
\hline Erythroderma & $\begin{array}{l}\text { Scabbed keratosis and } \\
\text { diffuse pigmented } \\
\text { pruriginous lesions }\end{array}$ & Not reported & $\begin{array}{l}\text { Fixed patches and } \\
\text { lesions mainly } \\
\text { photodistributed }\end{array}$ & $\begin{array}{l}\text { Fixed patches and } \\
\text { lesions mainly } \\
\text { photodistributed }\end{array}$ & Fixed patches and lesions \\
\hline $\begin{array}{l}\text { Infiltrating lymphocytic } \\
\text { phenotype }\end{array}$ & CD8+ T-lymphocytes & CD8+ T-lymphocytes & CD4+ T-lymphocytes & CD4+ T-lymphocytes & CD4+ T-lymphocytes \\
\hline Treatment & HAART & HAART & $\begin{array}{l}\text { Radiation therapy, } \\
\text { topical corticosteroids }\end{array}$ & $\begin{array}{l}\text { Photochemotherapy, } \\
\text { topical } \\
\text { chemotherapy }\end{array}$ & $\begin{array}{l}\text { Multiagentchemotherapy } \\
\text { often married to } \\
\text { interferons }\end{array}$ \\
\hline
\end{tabular}

ACLD = atypical cutaneous lymphoproliferative disorder; DILS = diffuse infiltrative lymphocytosis syndrome; CTCL: MF = cutaneous T-cell lymphoma: Mycosis Fungoides. SS = Sézary's Syndrome; ATLL = adult T-cell lymphoma/leukemia, and HAART = highly active antiretroviral therapy. 


\section{Conclusion}

We alert healthcare providers to this rare HIV-associated condition. The broad differential of severe and diffuse erythroderma in the backdrop of HIV infection, the problematic and potentially life-threatening nature of severe and diffuse ACLD, the stereotypical changes in peripheral blood T-lymphocyte subsets in absence of an obvious clonal disorder of lymphocytes, the potential beneficial effect of HAART and alternative options to treat ACLD are central points to note.

\section{REFERENCES}

[1] H. Bachelez, F. Hadida, C. Parizot, et al., "Oligoclonal Expansion of HIV-Specific Cytotoxic CD8 T Lymphocytes in the Skin of HIV-1-Infected Patients with Cutaneous Pseudolymphoma," The Journal of Clinical Investigation, Vol. 101, No. 11, 1998, pp. 2506-2516. doi:10.1172/JCI1450

[2] E. Sbidian, M. Battistella, J. Rivet, H. Bachelez, et al., "Remission of Severe CD8+ Cytotoxic T Cell Skin Infiltrative Disease in Human Immunodeficiency Virus-Infected Patients Receiving Highly Active Antiretroviral Therapy," Clinical Infectious Diseases, Vol. 51, No. 6, 2010, pp. 741-748. doi:10.1086/655895

[3] T. A. Longacre, K. Foucar, F. Koster and W. Burgdorf, "Atypical Cutaneous Lymphoproliferative Disorder Resembling Mycosis Fungoides in AIDS," The American Journal of Dermatopathology, Vol 11, No. 5, 1989, pp. 451-456. doi:10.1097/00000372-198910000-00007

[4] S. Itescu, J. Dalton, H. Z. Zhang and R. Winchester, "Tissue Infiltration in a CD8 Lymphocytosis Syndrome Associated with Human Immunodeficiency Virus-1 Infection Has the Phenotypic Appearance of an Antigenically Driven Response,” Journal of Clinical Investigation, Vol. 91, No. 5, 1993, pp. 2216-2225. doi:10.1172/JCI116448

[5] R. G. Egbers, T. T. Do, L. Su, Y. R. Helfrich, J. E. Gudjonsson, "Rapid Clinical Change in Lesions of Atypical Cutaneous Lymphoproliferative Disorder in an HIV Patient: A Case Report and Review of the Literature,” Dermatology Online Journal, Vol. 17, No. 9, 2011, p. 4.

[6] J. R. Golbus, G. Gallagher, G. Blackburn and S. Cinti, "Polyneuropathy Associated with the Diffuse Infiltrative Lymphocytosis Syndrome,” Journal of the International Association of Providers of AIDS Care, Vol. 11, No. 4, 2012, pp. 223-226. doi:10.1177/1545109712442798

[7] D. Basu, F. M. Williams, C. W. Ahn and J. D. Reveille, "Changing Spectrum of the Diffuse Lymphocytosis Syndrome," Arthritis Care and Research, Vol. 55, No. 3, 2006, pp. 466-472. doi:10.1002/art.21980

[8] P. F. Levay and M. E. Motes, "Diffuse Infiltrative Lymphocytosis Syndrome (DILS)," South African Family Practice, Vol. 50, No. 2, 2008, pp. 42-44.

[9] S. Friedler, M. T. Parisi, E. Waldo, R. Wieczorek, G. Sidhu and M. J. Rico, “Atypical Cutaneous Lymphopro- liferative Disorder in Patients with HIV Infection,” International Journal of Dermatology, Vol. 38, No. 2, 1999, pp. 111-118. doi:10.1046/j.1365-4362.1999.00417.x

[10] W. D. James, R. R. Redfield, G. P. Lupton, et al., “A Papular Eruption Associated with Human T Cell Lymphotropic Virus Type III Disease,” Journal of the American Academy of Dermatology, Vol. 13, No. 4, 1985, pp. 563-566. doi:10.1016/S0190-9622(85)70197-1

[11] N. S. Sadick and N. S. McNutt, "Cutaneous Hypersensitivity Reactions in Patients with AIDS,” International Journal of Dermatology, Vol. 32, No. 9, 1993, pp. 621-627. doi:10.1111/j.1365-4362.1993.tb04014.X

[12] M. R. Buchness, H. W. Lim, V. A. Hatcher, et al., "Eosinophilic Pustular Folliculitis in the Acquired Immunodeficiency Syndrome," The New England Journal of Medicine, Vol. 318, No. 18, 1988, pp. 1183-1186. doi:10.1056/NEJM198805053181807

[13] S. A. Coopman, R. A. Johnson, R. Platt and R. S. Stern, "Cutaneous Disease and Drug Reactions in HIV Infection,” The New England Journal of Medicine, Vol. 328, No. 23, 1993, pp. 1670-1674. doi:10.1056/NEJM199306103282304

[14] T. Nagatani, "Adult T-Cell Leukemia/Lymphoma Cutaneous Type,” International Journal of Dermatology, Vol. 35, No. 6, 1996, pp. 400-401. doi:10.1111/j.1365-4362.1996.tb03018.x

[15] F. Gahongayire, "Mycosis Fungoides and Sézary Syndrome against a Human Immunodeficiency Virus-Positive Background: Case Report,” International Journal of Dermatology, Vol. 46, No. 1, 2007, pp. 32-35.

[16] R. Willemze, "Primary Cutaneous Lymphomas," Annals of Oncology, Vol. 22, No. 4, 2011, pp. iv72-iv75. doi:10.1093/annonc/mdr181

[17] E. A. Kotz, D. Anderson and B. H. Thiers, "Cutaneous T-Cell Lymphoma,” Journal of the European Academy of Dermatology and Venereology, Vol. 17, No. 2, 2003, pp. 131-137. doi:10.1046/j.1468-3083.2003.00635.x

[18] T. Takahashi, H. Tsukuda, H. Itoh, M. Tsujisaki, et al., "Primary and Isolated Adult T-Cell Leukemia/Lymphoma of the Bone Marrow," The Japanese Society of Internal Medicine, Vol. 50, No. 20, 2011, pp. 2393-2396. doi:10.2169/internalmedicine.50.5857

[19] J. J. Campbell, R. A. Clark, R. Watanabe and T. S. Kupper, "Sézary Syndrome and Mycosis Fungoides Arise from Distinct T-Cell Subsets: A Biologic Rationale for Their Distinct Clinical Behaviors,” Blood, Vol. 116, No. 5, 2010, pp. 767-771. doi:10.1182/blood-2009-11-251926

[20] K. Takatsuki, "Discovery of Adult T-Cell Leukemia," Retrovirology, Vol. 2, No. 16, 2005, pp. 16-18.

[21] M. Duvic, M. Donato, B. Dabaja, C. Hosing, et al., "Total Skin Electron Beam and Non-Myeloablative Allogeneic Hematopoietic Stem-Cell Transplantation in Advanced Mycosis Fungoides and Sézary Syndrome,” Journal of Clinical Oncology, Vol. 28, No. 14, 2010, pp. 2365-2372. doi:10.1200/JCO.2009.25.8301

[22] M. Janier, C. Katlama, B. Flageul, et al., "The Pseudo-Sézary-Syndrome with CD8 Phenotype in a Patient 
with the Acquired Immunodeficiency Syndrome (AIDS)," Annals of Internal Medicine, Vol. 110, No. 9, 1989, pp. 738-740.

[23] P. Zhang, L. Chiribogy, M. Jacobsen, et al., "Mycosis Fungoides-Like T-Cell Cutaneous Lymphoid Infiltrates in Patients with HIV Infection,” American Journal of Dermatopathology, Vol. 17, No. 1, 1995, pp. 29-35.

[24] H. Bachelez, F. Hadida and G. Gorochov, "Massive Infiltration of the Skin by HIV-Specific Cytotoxic CD8+
Cells,” The New England Journal of Medicine, Vol. 335, No. 1, 1996, pp. 61-62. doi:10.1056/NEJM199607043350118

[25] M. E. Moreno-Fernandez, W. Zapata, J. T. Blackard, G. Franchini and C. A. Chougnet, "Human Regulatory T Cells Are Targets for Human Immunodeficiency Virus Infection, and Their Susceptibility Differs Depending on the HIV Type 1 Strain,” Journal of Virology, Vol. 83, No. 24, 2009, pp. 12925-12933. doi:10.1128/JVI.01352-09 